\title{
Lack of Association between MDR1 G2677T/A Polymorphism and Leukemia Risk: An Updated Meta-Analysis
}

\author{
Limin Ma Haiping Yang Linhai Ruan \\ Department of Hematology, The First Affiliated Hospital, Henan University of Science and Technology, Luoyang, China
}

\section{Keywords}

Meta-analysis · Multidrug resistance gene .

Polymorphism - Leukemia - Genetic susceptibility

The multidrug resistance gene (MDR1) encodes P-glycoprotein (P-gp), a member of the ATP-binding cassette (ABC) superfamily. $\mathrm{P}$-gp functions as an efflux pump in an ATP-dependent way, which transportes exogenous and endogenous substrates from the inside of cells to the outside and provides a defense mechanism against potentially harmful xenobiotics [1]. Alteration of P-gp activity results in decreased extrusion of harmful compounds and cumulative cytotoxicity, which has been associated with the development of various cancers [2]. More than 50 single nucleotide polymorphisms (SNPs) have been identified in the MDR1 gene. G2677T/A at exon 21(rs2032582) is the most frequent SNP in the coding region of $M D R 1$ and has been related to the altered $\mathrm{P}$-gp expression level and transport activity [3, 4]. Therefore, the MDR1 G2677T/A variant may play a role in the etiology of cancers. Several studies have investigated the relationship between MDR1 G2677T/A polymorphism and the risk for leukemia, but the results were controversial. Therefore, a meta-analysis was performed to synthetically evaluate the association.

The PubMed, Elsevier, EMBASE, China National Knowledge Infrastructure platform and Wanfang databases were searched (until May 2014) to identify studies that assess the relationship between MDR1 G2677T/A polymorphism and leukemia risk. The search terms included: 'MDR1' OR 'multidrug resistance gene' OR 'ABCB1', 'polymorphism' OR 'mutant' OR 'variant', AND 'leukemia' OR 'leukaemia' OR 'leukocythemia'. The references of the eligible studies were also reviewed to find additional studies. The following inclusion criteria were used for study selection: (i) published literature in English or Chinese; (ii) case-control designed studies; (iii) genotype frequencies for both cases and controls were available. 2 authors examined the retrieved literature independently and disagreement was resolved by consensus. The quality of the included studies was evaluated using the Newcastle-Ottawa Scale. A maximum score of 9 points could be assigned, where studies with 6 or more points were considered high-quality studies. The pooled odds ratios and $95 \%$ confidence intervals were used to assess the strength of association. Totally, 9 studies bearing 1,389 cases and 1,823 controls were included in the meta-analysis. Supplemental table 1 ( $w w w$. karger.com/?DOI=435904) summarizes the main characteristics of the included studies. In overall comparisons, there was no significant association between MDR1 G2677T/A polymorphism and leukemia risk under 4 genetic models. In the stratification analyses by ethnicity, age, leukemia subtype, and control source, no significant association was observed in any subgroup. The main results of quantitative synthesis are listed in supplemental table 2 ( $w w w . k a r g e r . c o m / ? D O I=435904)$. Sensitivity analysis was conducted by omission of studies not in HardyWeinberg equilibrium, and the combined results were not statistically significantly changed, which showed the relative stablity and credibility of the results of the present meta-analysis. A funnel plot was applied to evaluate the publication bias and the results showed that all points in the funnel plot were distributed symmetrically, revealing no statistical evidence for publication bias.

In this report, we found no significant association between MDR1 G2677T/A polymorphism and the risk for leukemia in the overall population and the stratification analyses. The results of this meta analysis were not in line with the study reported by Yan et al. [5], in which a significant association was found in Asians and Africans and myeloid leukemia, suggesting that G2677T polymorphism might be a protective factor in the susceptibility to myeloid leukemia. Our results are more convincing because of the relatively lager sample size. However, some limitations should be kept in mind. First, subgroup analyses could not be conducted in Africans and mixed population due to the limited number of

\section{KARGER}

() 2015 S. Karger GmbH, Freiburg

Fax +497614520714 
included studies, and the results were mainly applicable to Asian and Caucasian ethnicities. Second, for lack of sufficient data, we were unable to evaluate potential correlative factors such as environmental exposures and lifestyle which have an important impact on leukemia genesis. In addition, a relatively small number of eligible studies were included in this meta-analysis, which may have insufficient statistical power to detect possible associations.

In summary, the present meta-analysis revealed that G2677T/A polymorphism in MDR1 was not associated with leukemia risk. However, well-designed studies with lager sample size are needed to confirm our findings.

\section{Supplemental Material}

Supplemental Table 1. The main characteristics of included case-control studies

Supplemental Table 2. Results of quantitative synthesis for MDR1 G2677T/A polymorphism with leukemia risk

To access the supplemental tables please refer to www.karger.com/? DOI $=435904$.

\section{Disclosure Statement}

The authors declare that they have no conflicts of interest.

\section{References}

1 Hodges LM, Markova SM, Chinn LW, et al.: Very important pharmacogene summary: ABCB1 (MDR1, P-glycoprotein). Pharmacogenet Genomics 2011;21: 152-161.

2 Brambila-Tapia AJ: MDR1 (ABCB1) polymorphisms functional effects and clinical implications. Rev Invest Clin 2013;65:445-454.
Lamba J, Strom S, Venkataramanan R, et al.: MDR1 genotype is associated with hepatic cytochrome $\mathrm{P} 450$ 3A4 basal and induction phenotype. Clin Pharmacol Ther 2006;79:325-338.

4 Kurata Y, Ieiri I, Kimura M, et al.: Role of human MDR1 gene polymorphism in bioavailability and interaction of digoxin, a substrate of P-glycoprotein. Clin Pharmacol Ther 2002;72:209-219.
Yan Y, Liang H, Xie L, et al.: Association of MDR1 G2677T polymorphism and leukemia risk: evidence from a meta-analysis. Tumour Biol 2014;35:21912197. 